\title{
White-line evolution in shocked solid Ta evidenced by synchrotron x-ray absorption spectroscopy
}

\author{
C. M. Pépin, ${ }^{1,2}$ R. Torchio,${ }^{3}$ F. Occelli, ${ }^{1,2}$ E. Lescoute, ${ }^{1,2}$ O. Mathon, ${ }^{3}$ V. Recoules, ${ }^{1,2}$ J. Bouchet,${ }^{1,2}$ L. Videau,,${ }^{1,2}$ \\ A. Benuzzi-Mounaix ${ }^{4}$ T. Vinci, ${ }^{4}$ R. Briggs,,${ }^{3,}$ S. Pascarelli, ${ }^{3}$ R. Gaal, ${ }^{5}$ P. Loubeyre,,${ }^{1,2}$ and A. Sollier ${ }^{1,2}$ \\ ${ }^{1} C E A, D A M, D I F, 91297$ Arpajon, France \\ ${ }^{2}$ Université Paris-Saclay, CEA, Laboratoire Matière en Conditions Extrêmes, 91680 Bruyères-le-Châtel, France \\ ${ }^{3}$ ESRF, 6 Rue Jules Horowitz BP220, F-38043 Grenoble Cedex, France \\ ${ }^{4}$ Laboratoire d'Utilisation des Lasers Intenses - CNRS, Ecole Polytechnique, CEA, Université Paris-Saclay, F-91128 Palaiseau Cedex, France \\ ${ }^{5}$ Earth and Planetary Science Laboratory, Institute of Physics, Ecole Polytechnique Fédérale de Lausanne, CH-1015 Lausanne, Switzerland
}

\begin{abstract}
Time-resolved x-ray absorption experiments have been performed on shocked tantalum. Using a single 100 ps synchrotron x-ray pulse, nanosecond-lived equilibrium states of shocked solid Ta have been measured by $L_{3}$-edge absorption spectroscopy. The energy shift and intensity change of the white line were measured up to $130 \mathrm{GPa}$ and $2000 \mathrm{~K}$. The experimental results are discussed in light of density functional theory calculations and FEFF simulations. The absence of occurrence of the hcp- $\omega$ phase is suggested from the analysis of the shape of the white line.
\end{abstract}

\section{INTRODUCTION}

Tantalum (Ta) is a model body-centered-cubic (bcc) metal of high stability with increasing pressure and temperature. The simple bcc structure simplifies the description of the mechanical response of Ta metal under stress. Furthermore Ta is a refractory metal, with a melting temperature among the highest for a material, thus making it an important metal for applications. The phase diagram of Ta and its properties under pressure have been extensively studied. In particular, there exist ongoing experimental and theoretical contentions regarding the melting curve and the polymorphism of Ta at high pressure [1-7]. A few years ago, measurements of Ta melting points under static condition in a laser-heating diamond anvil cell were showing a flat melting curve and a puzzling large discrepancy with shock wave data and ab initio calculations. That melting temperature difference, spreading over a few $1000 \mathrm{~K}$ in the $100 \mathrm{GPa}$ range [1,2], was resolved when a microscopic signature of melting based on synchrotron $\mathrm{x}$-ray diffraction (XRD) was implemented [3]. Furthermore, the bcc phase was shown stable up to melting and $120 \mathrm{GPa}$. However, other measurements using a flash-heating technique gave melting points still below calculations and shock wave data [4]. In order to explain this apparent discrepancy between the static and dynamic melting curve, it was suggested that prior to melting Ta will undergo a shear-induced phase transition and so the melting point in dynamical compression could be higher. The possible phase transition in Ta under deviatoric stress is actively discussed. Burakovsky et al. have now

\footnotetext{
${ }^{*}$ Present address: Lawrence Livermore National Laboratory, Livermore, CA 94550, USA.

†arnaud.sollier@cea.fr
}

suggested that tantalum could transform from the bcc phase to the hcp- $\omega$ phase at $\sim 70 \mathrm{GPa}$ [7]. This prediction was further supported by sound speed measurements in shocked Ta showing a kink at $\sim 60 \mathrm{GPa}$ [8]. Moreover, two recovery experiments on Ta samples shocked to $45 \mathrm{GPa}$ with a $1.8 \mu \mathrm{s}$ load duration and shocked to $70 \mathrm{GPa}$ with a $3.7 \mathrm{~ns}$ laser pulse showed a small amount of hcp- $\omega$ phase in recovered Ta [9-12]. On the other hand, recent calculations $[13,14]$ have shown that the hcp- $\omega$ phase of Ta is energetically unfavorable and mechanically unstable at high pressure and high temperature. Also, recent sound speed measurements $[15,16]$ have found no evidence of a phase transition up to the shock melting pressure of Ta. Experiments to test the existence of a phase transition in tantalum before melting under shock compression are still needed. The possibility of inspecting the actual atomistic arrangement of material under dynamical compression offers a good opportunity to obtain unambiguous answers. Recently, using synchrotron nanosecond time-resolved XRD on laser-shocked $\mathrm{Bi}$, significant deviations between phase diagrams obtained under static and dynamical compressions, respectively, could be disclosed [17]. Here, synchrotron nanosecond time-resolved $\mathrm{x}$-ray absorption fine structure (XAFS) measurements are performed to investigate at the microscopic level the properties of shocked Ta. XAFS provides information on both the electronic structure and the local order. Measurements have been performed at the Ta $L_{3}$ edge $(9.881 \mathrm{keV})$ which is associated with a direct-dipole transition, hence with the appearance of a strong peak just above the absorption edge called the white line (WL). The shift and shape of the white line, directly related to the shift of the atomic levels and the evolution of the electronic density of state, are compared to $a b$ initio calculations. The $\mathrm{x}$-ray absorption near-edge structure (XANES) spectra are also analyzed to look for a signature of the hcp- $\omega$ phase. 


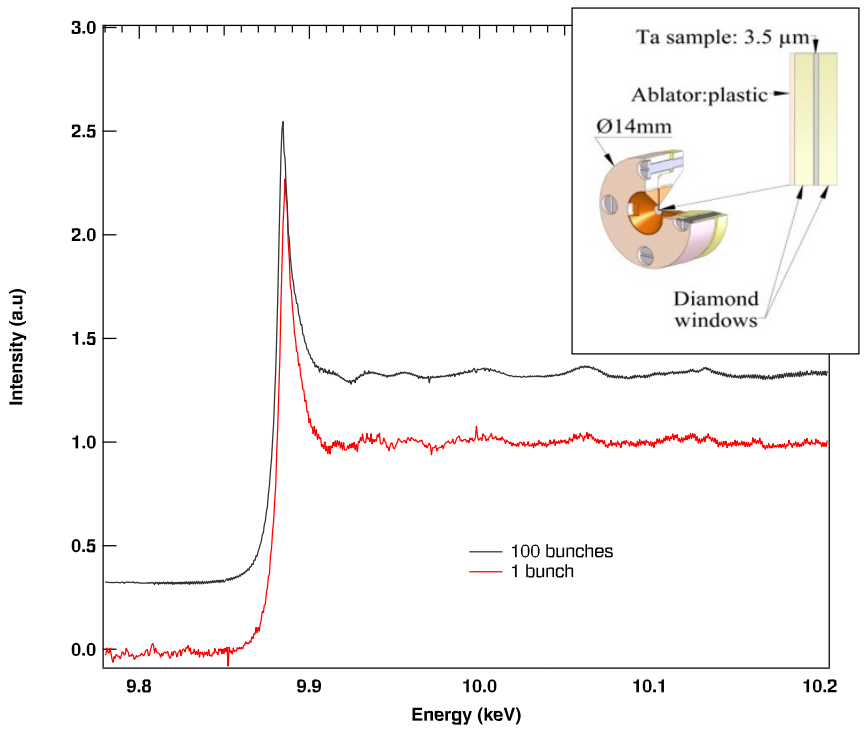

FIG. 1. Comparison of EXAFS spectra recorded with 1 and 100 bunches on the same sample. Inset: Schematic of the target holder and assembly.

\section{METHODS}

\section{A. Experimental configuration}

The pump-probe experiments were performed at the European Synchrotron Research Facility (ESRF) on the $L$ branch of the energy-dispersive $\mathrm{x}$-ray absorption spectroscopy (EDXAS) beamline ID24 [18,19]. For our experiment, the horizontal focusing of the $\mathrm{x}$ ray was performed by an elliptically bent $\mathrm{Si}(111)$ polychromator in Bragg geometry allowing us to reach horizontal focal spots down to $5 \mu \mathrm{m}$. In this geometry, the pink x-ray source is set on one of the foci of the ellipse and the sample is placed on the second one (focal spot). The x-ray beam energy, diffracted by the crystal, varies continuously from one side to the other side of the crystal, and a correlation can be established between the propagation direction of the x-ray beam and its energy. The beam transmitted by the sample is then detected by a position-sensitive detector allowing the simultaneous collection of the whole spectrum, covering a range up to $300 \mathrm{eV}$ above the Ta $L_{3}$ edge $(9.881 \mathrm{keV})$. Each pixel detects a narrow energy bandwidth of the incoming polychromatic x-ray beam, transforming the energy/direction correlation into an energy/position correlation. Data were acquired using a single x-ray pulse of 100 ps duration, during a 4-bunch mode configuration of the synchrotron storage ring that provides the highest number of photons per pulse $(\sim 10 \mathrm{~mA}$ bunch) and a maximum separation between pulses (700 ns). Examples of extended XAFS (EXAFS) spectra on Ta recorded with one pulse and with 100 pulses are shown in Fig. 1. The XH position-sensitive microstrip-based detector [19,20], equipped with $1024 \mathrm{Ge}$ pixels, was operated with an integration time of $680 \mathrm{~ns}$ and phase locked to the ESRF radio-frequency system, ensuring integration of the signal generated by a unique bunch of electrons in the storage ring. The vertical focusing was performed by a pair of mirrors in a Kirkpatrick-Baez configuration [18]. With this setup, the $\mathrm{x}$-ray spot could be focused down to
$5 \times 10 \mu \mathrm{m}$ FWHM $(H \times V)$ to ensure that the probed part of the compressed sample was not perturbed by edge effects. The laser was focused on the target under vacuum as described previously in the shocked Fe XAS experiment [21]. The laser beam provided by the Générateur de Chocs Laser Transportable (GCLT) portable high-power laser system was focused under vacuum on the target with an angle of $30^{\circ}$, launching an ablation-driven compression in the target perpendicular to its surface. All the experiments were performed at a wavelength of $1053 \mathrm{~nm}$ with $10 \mathrm{~ns}$ FWHM square laser pulses and a maximum energy of $30 \mathrm{~J}$. The energy, pulse duration, and near-field spatial profile of the laser pulse were measured for all shots. The x-ray beam probed the shocked Ta parallel to the compression axis with variable drive-probe delays. The delay between the laser and the X-ray pulse was adjusted using a fast Si APD photodiode (Hamamatsu S2383). A fine temporal alignment was also performed by measuring the onset of the bcc-hcp transition in pure Fe targets, that shows up in clear XAS modifications. The optimization of the compressed volume of Ta required a careful target design that was optimized using two hydrocodes, the ESTHER 1D code [22] and the MULTI 2D code [23]. The target was made of a thin $(3.5 \mu \mathrm{m})$ deposit of pure Ta sandwiched between two diamond windows $(96 \%$ transmission at $9.9 \mathrm{keV}$ for $50 \mu \mathrm{m}$ thickness), which act to confine a homogeneous shocked state over a few-ns time interval, i.e., much longer than the 100 ps x-ray pulse. A $\mathrm{CH}$ layer was deposited on the ablation surface to limit preheating effects. Two different kinds of targets were prepared to achieve different thermodynamic states. In the thick-target package used for low-pressure experiments, the front and rear diamond windows were $40 \mu \mathrm{m}$ and $50 \mu \mathrm{m}$ thick, respectively. In the thin-target package used for the high-pressure experiments, both diamond windows had a thickness of $25 \mu \mathrm{m}$. Each target was first carefully inserted inside a $14 \mathrm{~mm}$ diameter brass holder (Fig. 1, inset) in order to make its handling easier. Next, the brass holder was screwed inside a rotative target wheel that contained 20 target packages. This specific support system allowed us to ensure a reproducible target positioning inside the vacuum chamber along the x-ray path. The alignment of the x-ray beam and the laser beam was performed in two steps, using high-magnification microscopes located on both sides of the target. First, the x-ray beam was aligned on a pinhole drilled inside a thin metallic foil inserted in one of the brass holders. The focal spot of the laser beam was then moved until it matched perfectly with the $\mathrm{x}$-ray position, and this position was referenced on our two visualization screens as the reference position. The metallic foil was finally replaced by a target package, and if necessary, the position of the laser was slightly corrected to compensate the focus difference related to the thickness difference between the foil and the target package. The time $t=0$ corresponds to when the laser drive is incident upon the ablation layer. The various parameters of the different experimental configurations corresponding to the four runs are summarized in Table I.

In the first run, thick targets were used and the laser was focused with a $250 \mu \mathrm{m}$ diameter phase plate producing a maximum usable laser intensity of $3.5 \times 10^{12} \mathrm{~W} / \mathrm{cm}^{2}$ corresponding to a maximum pressure of $\sim 60 \mathrm{GPa}$ in Ta. The shock launched in the front window generates reverberating 
TABLE I. Target, $\mathrm{x}$-ray, and laser parameters used in our four runs of experiments.

\begin{tabular}{lccccr}
\hline \hline Run number & Target package & $\begin{array}{c}\text { X-ray focal spot } \\
(H \times V) \text { in } \mu \mathrm{m}\end{array}$ & $\begin{array}{c}\text { Phase plate diameter } \\
(\mu \mathrm{m})\end{array}$ & $\begin{array}{c}\text { On-target irradiance } \\
\left(\mathrm{W} / \mathrm{cm}^{2}\right)\end{array}$ & Expected $P(\mathrm{GPa})$ and $T(\mathrm{~K})$ \\
\hline Run 1 & Thick & $9 \times 14$ & 250 & $3.5 \times 10^{12}$ & $60 \mathrm{GPa}, 700 \mathrm{~K}$ \\
Run 2 & Thick & $9 \times 14$ & 100 & $2.1 \times 10^{13}$ & $2.1 \times 10^{13}$ \\
Run 3 & Thin & $6 \times 8$ & 100 & $3.3 \times 10^{13}$ & $130 \mathrm{GPa}, 2000 \mathrm{~K}$ \\
Run 4 & Thin & $6 \times 8$ & No & $150 \mathrm{GPa}, 2100 \mathrm{~K}$ \\
\hline \hline
\end{tabular}

compression waves in the tantalum foil due to the presence of the rear diamond window. Because of the impedance mismatch between diamond and tantalum, a succession of re-shocks are imparted to the tantalum and the final state of tantalum is therefore below its principal Hugoniot. The confinement time of the stable thermodynamic conditions in Ta was estimated by hydrodynamic simulations (Fig. 2) and measured directly from the modifications in the XANES spectra for various delays between the laser arrival on the target and the $\mathrm{x}$-ray pulse. As seen in Fig. 2 a -3 ns confinement time is observed during which thermodynamic conditions are stable. The pressure was estimated by hydrosimulations using the MULTI [23] and ESTHER [22] codes and also doublechecked using VISAR measurements done on a bare tantalum foil and on a thick target to correctly estimate the amount of laser energy deposited. The two hydrodynamic codes give consistent results. The reproducibility of the measured spectra was good. We are thus confident that homogeneous welldefined compressed states of Ta were measured.

In order to achieve pressures in the $120 \mathrm{GPa}$ range where the hcp- $\omega$ phase is expected to be stable, the $250 \mu \mathrm{m}$ phase plate was replaced by a smaller $100 \mu \mathrm{m}$ phase plate. To maintain a homogeneous thermodynamic state and limit 2D effects, Ta targets with $25 \mu \mathrm{m}$ thick diamond windows were used in that case. The $2 \mathrm{D}$ simulations give a $50 \mu \mathrm{m}$ diameter homogeneous region, larger than the $\mathrm{X}$-ray probe beam [21]. The x-ray focal spot was then optimized down to $5 \times 10 \mu \mathrm{m}$ $(H \times V)$ FWHM. Again, a homogeneous thermodynamic state could be probed; however, the reproducibility from shot to shot was poorer due to the request of a $10 \mu \mathrm{m}$ precision in alignment. As seen in Fig. 5, the EXAFS data quality obtained in this second configuration was as good as for the spectra acquired with the larger phase plate and with a larger x-ray spot.

\section{B. Ab initio calculations}

In order to qualitatively understand how the WL evolves under extreme conditions, simulations have been performed using the ab initio plane wave density functional theory (DFT) code ABINIT [24-27]. DFT is applied together with the generalized gradient approximation [28]. Simulations were performed in the framework of the projected augmented wave (PAW) method [29,30] using a PAW data set including

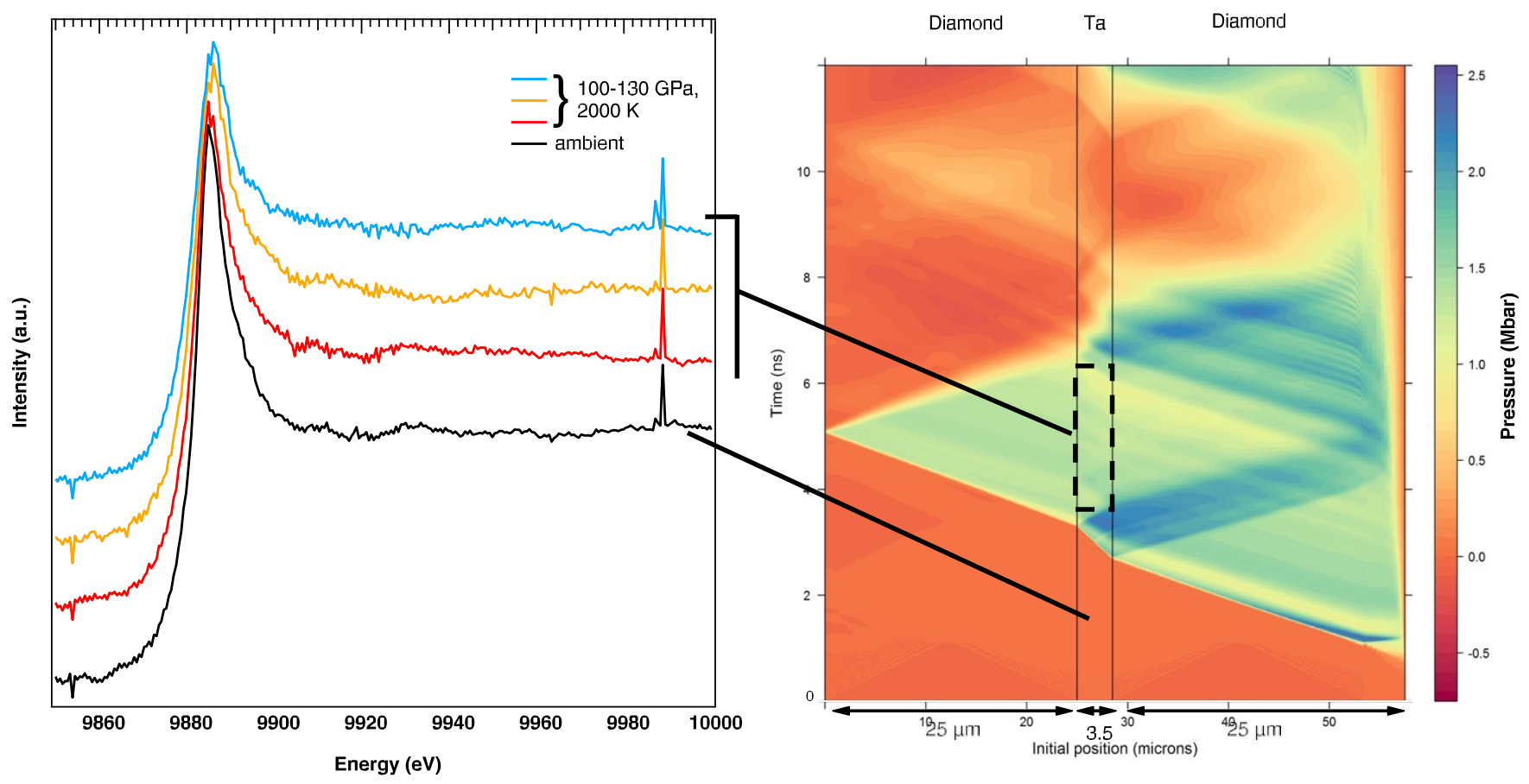

FIG. 2. Hydrodynamic simulation on a thin target package showing pressure as a function of time and position in the target. The laser is coming from the right (right panel). A quasi-homogeneous state is observed between 4 and 7 ns. Each x-ray absorption spectrum with a different color, as presented on the left, is acquired during this time window with an incremental time delay from the laser pulse. 


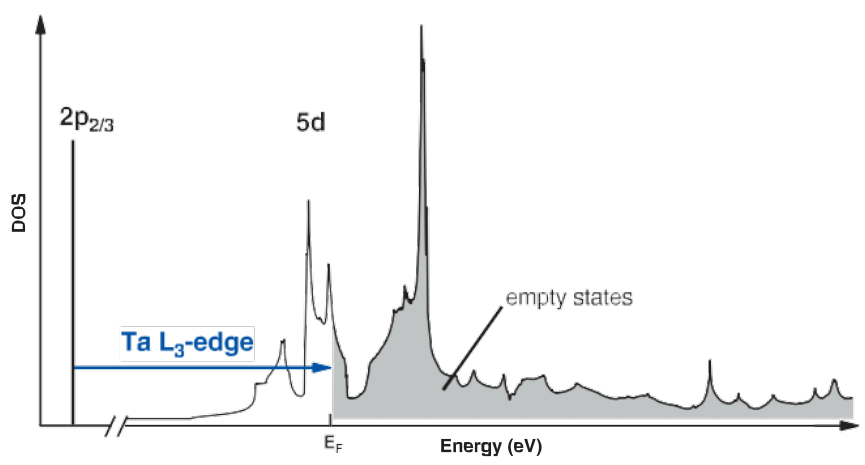

FIG. 3. Principle of $L_{3}$-edge XANES as a probe of the Ta $5 d$ band.

semicore states. Every PAW calculation needs several quantities defining the atomic species and the partial-wave basis for each type of atom. Such a set of parameters is called a PAW data set. The PAW data set for tantalum is generated using 13 outer electrons $\left(5 s^{2} 5 p^{6} 5 d^{3} 6 s^{2} 6 p^{0}\right)$ and a cutoff radius $r_{c}=1.21 a_{B}$. We use a plane wave basis with an energy cutoff of $25 \mathrm{Ha}$. The cubic simulation box includes 128 atoms for bcc and liquid phases, and 135 atoms for the $\omega$-hcp phase.

To simulate tantalum at a given thermodynamic state, molecular dynamic (MD) simulations were performed. At each ionic time step, forces are computed within DFT using the Hellman-Feynman theorem. The electron temperature $T_{e}$, which is controlled by the width of the Fermi-Dirac distribution, can be different from the ion temperature $T_{i}$. For nonequilibrium MD simulations, $T_{e}$ and $T_{i}$ are controlled independently. While $T_{e}$ is fixed for every time step in the
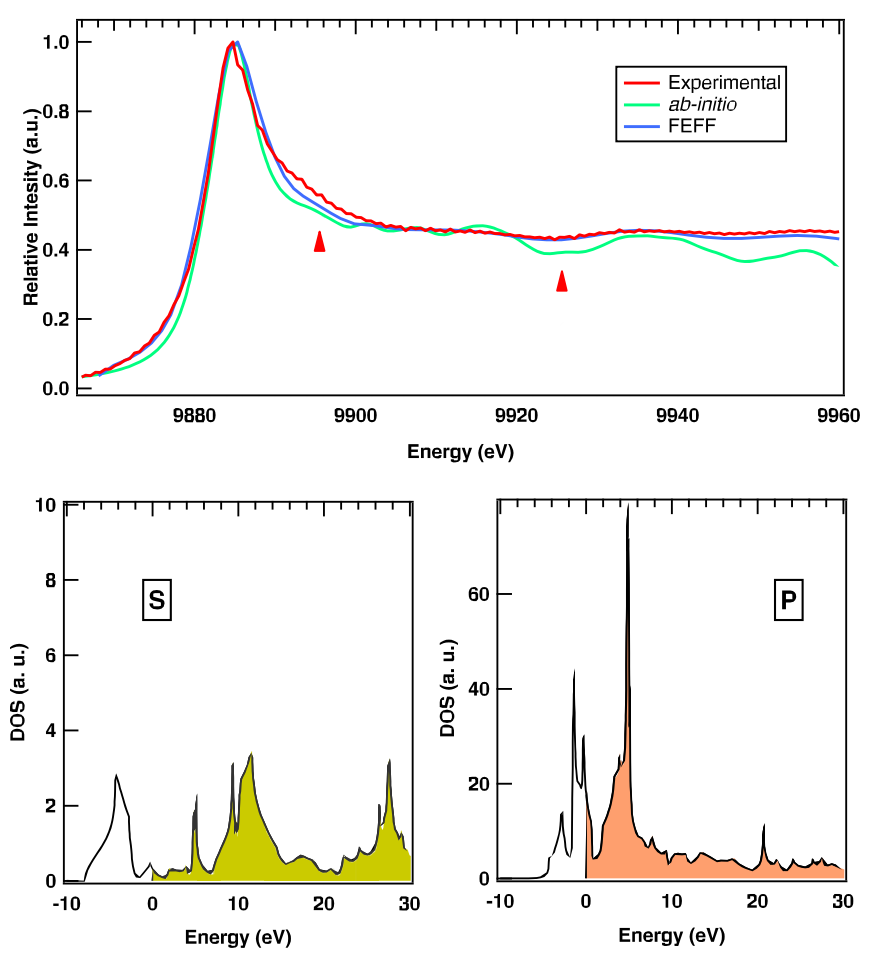

FIG. 4. Comparison of the experimental and calculated XANES spectra for tantalum near $L_{3}$ edge for ambient conditions (top). Calculated $s$ and $p$ projected DOS in the ambient conditions (bottom).
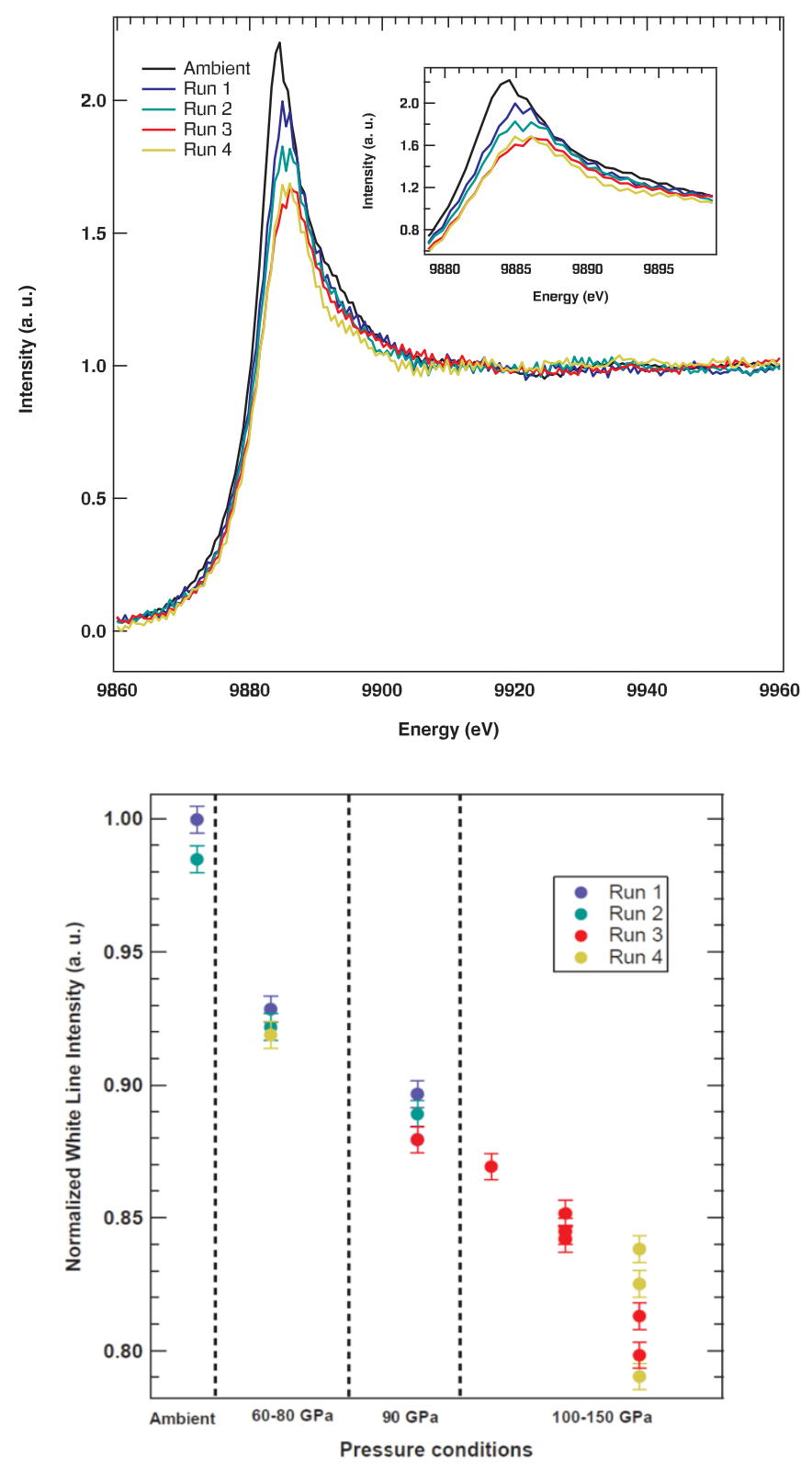

FIG. 5. Top: Maximum decrease of the white line observed for each run. Each spectrum corresponds to the highest $(P, T)$ conditions for each run. Bottom: Intensity of the WL normalized with ambient condition spectra as a function of the pressure conditions.

simulation, $T_{i}$ is controlled in the isokinetics ensemble where the velocity is rescaled at every time step to maintain the desired temperature. We started the simulation with 128 atoms initially arranged in an perfect bcc lattice. The equilibration for a given temperature is then monitored by looking at the pressure. Before reaching a stable ion structure, the system relaxed over a few hundred time steps associated with an increase of the pressure. After equilibration, the pressure fluctuated around a well-defined value and thermodynamic parameters as well as the $\mathrm{x}$-ray absorption spectra can then be obtained. The initial configuration is propagated up to 2 ps using time steps of $1.25 \mathrm{ps}$. All molecular dynamics calculations were performed at the $r$ point of the Brillouin zone. The $r$-point sampling is expected to be a good approximation 
for calculating the structure and dynamics of tantalum, but is not precise enough for accurate calculation of the electronic structure needed for the calculation of x-ray absorption spectra. The optical response in the $\mathrm{x}$-ray domain is then averaged over selected equally spaced ionic configurations along the equilibrated part of the trajectory. For each configuration, we run a DFT calculation with a $2 \times 2 \times 2$ Monkhorst-Pack grid and 5000 bands to obtain eigenstates converged up to $60 \mathrm{eV}$ above the edge. We then apply linear-response theory to obtain the absorption [31]. The absorption cross section for a given $\boldsymbol{k}$ point is expressed as

$$
\begin{aligned}
\sigma_{\boldsymbol{k}}(\omega)= & 4 \pi^{2} \omega \sum_{n}\left[1-f\left(\epsilon_{n, \boldsymbol{k}}\right)\right] \times\left|\left\langle\psi_{n, \boldsymbol{k}}|\vec{\nabla}| \phi_{\text {core }}\right\rangle\right|^{2} \\
& \times \delta\left(\epsilon_{\text {core }}-\epsilon_{n}-\hbar \omega\right) .
\end{aligned}
$$

We employ atomic units, with the electron charge $e$, Planck's constant $\bar{h}$, and the electron mass $m_{e}$ all set to unity. The $n$ summation ranges over the discrete bands (orbitals). $f\left(E_{n, \boldsymbol{k}}\right)$ is the Fermi-Dirac occupation factor corresponding to the energy $E_{n, \boldsymbol{k}}$ for the $n$th band for the $\boldsymbol{k}$ point $\boldsymbol{k}$. The total cross section is obtained by direct summation over all necessary $\boldsymbol{k}$ points. The core | $\boldsymbol{\varphi}_{\text {core }}>$ are obtained from the all-electron atomic calculation performed when the PAW data sets are generated using the Atompaw code [32]. The delta function in Eq. (1) is replaced by a Lorentzian function whose width is in general set to a tabulated value for the natural width of the core level. In our calculations, we use a fixed width of $4.89 \mathrm{eV}$ corresponding to the natural width for the $L_{3}$ edge of tantalum [33].

The absolute position of the edge cannot be formally obtained in the present model. The theoretical spectrum for room temperature was then shifted to match the experimental position of the edge. The same rigid shift was applied to all computed spectra.

The chemical potential is gbtained by the conservation of the number of electron $N=\int g(\varepsilon, T) f(\varepsilon, \mu, T)$, which changes with density. The energy of the core orbitals also moves with the density. To calculate the density dependence of the XANES spectra and $L_{3}$-edge shift, we then need to evaluate the displacement of the $2 p$ orbital as a function of density. In our DFT-PAW calculation, only valence electrons are explicitly treated during the calculation. Core electrons up to the $5 s$ are frozen. We need to go beyond this frozen core approximation. One possibility would be to generate the PAW data set for Ta keeping only $1 s$ and $2 s$ as core states. This kind of PAW data set requires a very high cutoff energy for the basis set and the calculation is not feasible. Instead, we used the all-electron DFT code WIEN2k $[34,35]$ to obtain the variation of the core orbitals as a function of the density. The simulation were performed for 1 atom in a cubic cell with bcc structure for several densities. We used the same exchange and correlation functional as in the ABINIT simulation (PBE [28]). The energy variation of the $2 p_{3 / 2}$ orbitals obtained in WIEN2k is used to obtain the shift of the edge for each density.

In addition, FEFF 9.6 [36] simulations were also performed over clusters of 65 and 71 atoms for the bcc and hep- $\omega$ phase at the estimated $P, T$ conditions. FEFF is an ab initio code for calculating excitation spectra and electronic struc- ture. It uses an $a b$ initio self-consistent real-space multiple scattering approach that can include polarization dependence, core-hole effects, and local-field corrections, based on self-consistent, spherical muffin-tin scattering potentials. Calculations are based on an all-electron, real-space relativistic Green's function formalism with no symmetry requirement. The code includes vibrational effects in terms of DebyeWaller factors. The XANES simulations reported here were performed using the Hedin-Lundqvist self-energy exchange correlation potential, with 15 iterations for the potential calculation for the self-consistency, and full multiple scattering radius of $6.3-7 \AA$. The core-hole interaction is treated using an RPA (random phase approximation) screening. Thermal effects are taken into account within the correlated-Debye method. An experimental broadening of $0.5 \mathrm{eV}$ was chosen to match the experimental white-line height at ambient conditions. The edge position is also not well defined in FEFF; therefore the simulated spectra have been shifted to the nominal edge energy value.

\section{XAS MEASUREMENTS}

XAFS refers to the details of how $\mathrm{x}$ rays are absorbed by an atom at energies near and above the core-level bind- ing energies of that particular atom [37]. X-ray absorption spectra are traditionally divided into two energy regions of interest: The first, termed x-ray absorption near-edge structure (XANES), occurs in the region from the edge to approximately $30 \mathrm{eV}$ above the edge, while the second, termed the extended $\mathrm{x}$-ray absorption fine structure (EXAFS) extends from $30 \mathrm{eV}$ to $1000 \mathrm{eV}$ above the edge. The XANES region provides information on both the electronic structure and the local atomic order, which can be strongly changed going from the ambient solid conditions to warm dense matter (WDM) [38]. XANES has been used already to characterize the evolution of electron and ion structures in the WDM regime in shock compressed and/or isochoric heated matter $[39,40]$. In particular, it has been shown that this technique might be used as a diagnostic of the electronic temperature [41-43]. EXAFS refers to the oscillatory modulations in x-ray absorption spectra above the absorption edge energy, which are generated by interference between photoelectron waves and scattering by neighbor atoms [37]. The frequency of EXAFS modulations is related to the nearest-neighbor distance, hence to the compression. The damping rate of the modulation at higher x-ray energies is set by the Debye-Waller factor $[44,45]$, from which the lattice temperature can be determined.

\section{A. Evolution of the white line}

Spectral features near the Ta $L_{3}$ edge are dominated by photoabsorption that couples the $2 p_{3 / 2}$ core level with vacant $5 d$ states [46], as shown in Fig. 3. As the $5 d$ band is highly localized in energy, this results in a strong absorption peak, the white line. As a consequence the intensity and position of this WL provide an interesting experimental test bed to compare with ab initio calculations. The calculated XANES spectra for the ambient conditions from ab initio calculations and FEFF calculations are presented in Fig. 4, compared to a 
reference experimental spectrum. The high density of states of the $d$ levels is clearly seen in the projected density of states. We can see that we have an overall good agreement between theoretical and experimental spectra. In particular, the shoulder on the right of the white line and the minimum at $45 \mathrm{eV}$ above the edge are well reproduced, as evidenced by red arrows in Fig. 4. However, the simulations present more features than the experimental spectrum. Both DFT calculations reproduce well the white line but the FEFF results are in better agreement with the experimental EXAFS spectra. $A b$ initio molecular dynamics and FEFF simulations were then performed at the estimated conditions attained during the runs.

Upon compression the EXAFS oscillations are lost because of thermal disorder as shown in Fig. 2. The evolution of the white line (WL) could be quantitatively analyzed. The results of our four runs of data collection are summarized in Fig. 5. Different maximum pressure and temperature conditions are achieved in the runs. The height of the WL is seen in Fig. 5(b) to decrease with the increasing $P-T$ conditions achieved on the Ta sample. Figure 5(b) shows the evolution of the WL for each shot as a function of delay. For each run we observe a similar trend: the WL tends to decrease down to a minimum as the delay increases. This height minimum is linked to the highest conditions of pressure and temperature reached for each run. It is found around $10 \mathrm{~ns}$ delay for the thick targets ( 40 microns of diamond as a front window) and around $7 \mathrm{~ns}$ for the thin targets ( 25 microns of diamond as a front window). No noticeable difference is observed between the third and the fourth runs. While in both runs the same target package was used, in the fourth run, no phase plate was used. As a result, larger 2D effects were generated inside the target. While the compression should be greater in this fourth run, these $2 \mathrm{D}$ effects tend to reduce the maximum pressure reached. It is interesting to note that the maximum of the WL exhibits very little shift toward higher energies [see Fig. 5(a) inset].

While the WL maximum intensity is slightly overestimated, the trend of the decrease of the WL for the bcc structure in ab initio calculations is comparable to what is experimentally observed, as can be seen in Fig. 6 . The intensity decrease observed for the WL is linked to the partial filling of the $5 d$ state as temperature increases. The XANES experimental data provide important constraints on the electronic treatment of the solid at high density and temperature within the DFT framework. In agreement with the experimental data, very little shift of the WL maximum is observed in both simulations. The $L_{3}$-edge shift is related to the energy difference $E_{F} E_{2 p}$ and reflects the modification of the core energy level and of the electron density. The very good reproduction of the relative edge shift by DFT simulations validates the specific methodology used to obtain it.

\section{B. XANES spectra: Search for the $\omega$ phase}

The XANES spectra for the bcc and the $\omega$ phase, calculated by FEFF for the highest experimental conditions, at first seem quite similar at the edge region as seen in Fig. 7. The experimental WL decrease due to the shock loading is well reproduced assuming either a bcc or $\omega$ phase, meaning that

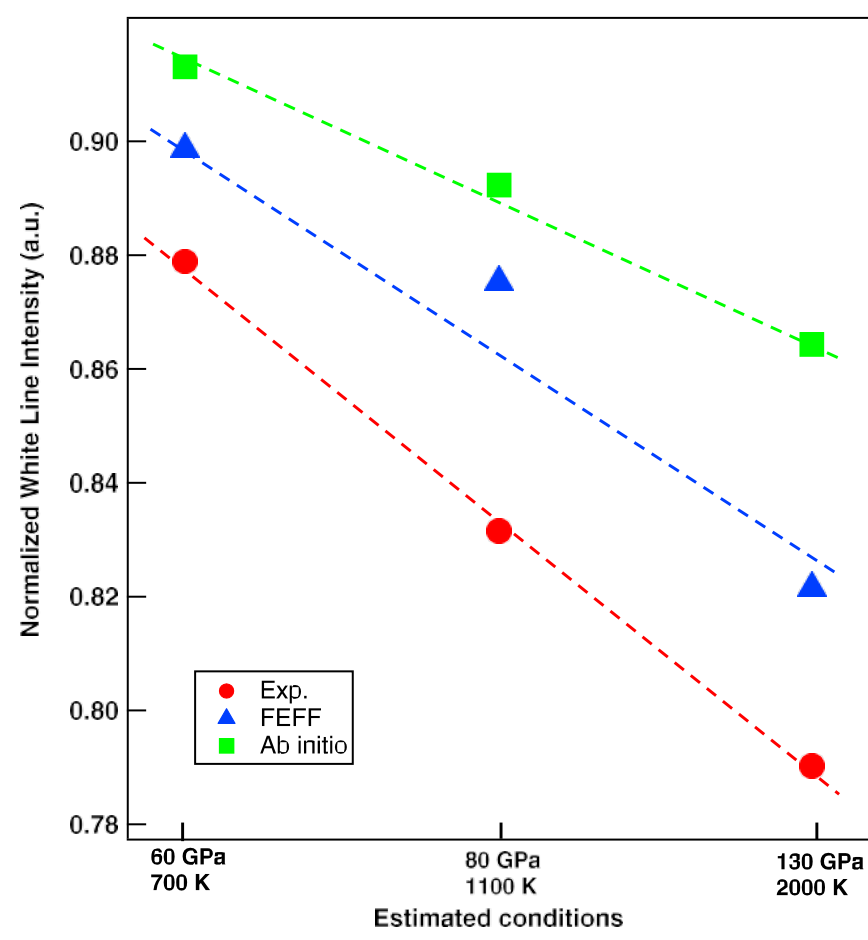

FIG. 6. Comparison of the experimental relative WL intensity with results of $a b$ initio for the different estimated conditions.

it cannot be used as a criterion to experimentally distinguish between them, although the $\omega$ phase gives a slightly less intense $\mathrm{WL}$ in the simulations. When considering the $\omega$ phase, the simulations show that it is associated with a broadening of the WL so that the WL of the $\omega$ phase crosses over the one from ambient conditions. We also note the appearance of a slight shoulder around $9882 \mathrm{eV}$. Both features are evidenced by the blue arrows in the inset of Fig. 7. These two features are not seen in the experimental data as shown by red arrows. The global shape of the experiment spectra seems thus better reproduced by the simulation of a bcc phase. However the noise levels for these ultrafast in situ measurements are too large to clearly rule out the appearance of the hcp- $\omega$ phase.

\section{CONCLUSION}

Time-resolved x-ray absorption spectroscopy was performed at the $L_{3}$ edge of tantalum under rapid laser compression. The number of photons in one bunch and the very good stability in energy of the dispersive geometry enable us to accurately measure the energy shift and intensity change of the strong absorption line, the white line. The WL intensity is observed to significantly decrease under compression. DFT and FEFF calculations follow a similar trend but the WL maximum intensity is overestimated. Probing the $L$-edge provides a stringent test for electronic calculations. For the XANES region the fact that many features cannot be easily distinguished depends mainly on the intrinsic energy resolution due to the core-hole lifetime at the $L$ edges. As shown, not many features in the ambient spectrum in the XANES part are seen. Hence, probing a direct dipole transition at the $L$ edge is more appropriate than probing the $K$ edge to investigate changes of electronic configurations through XAS 

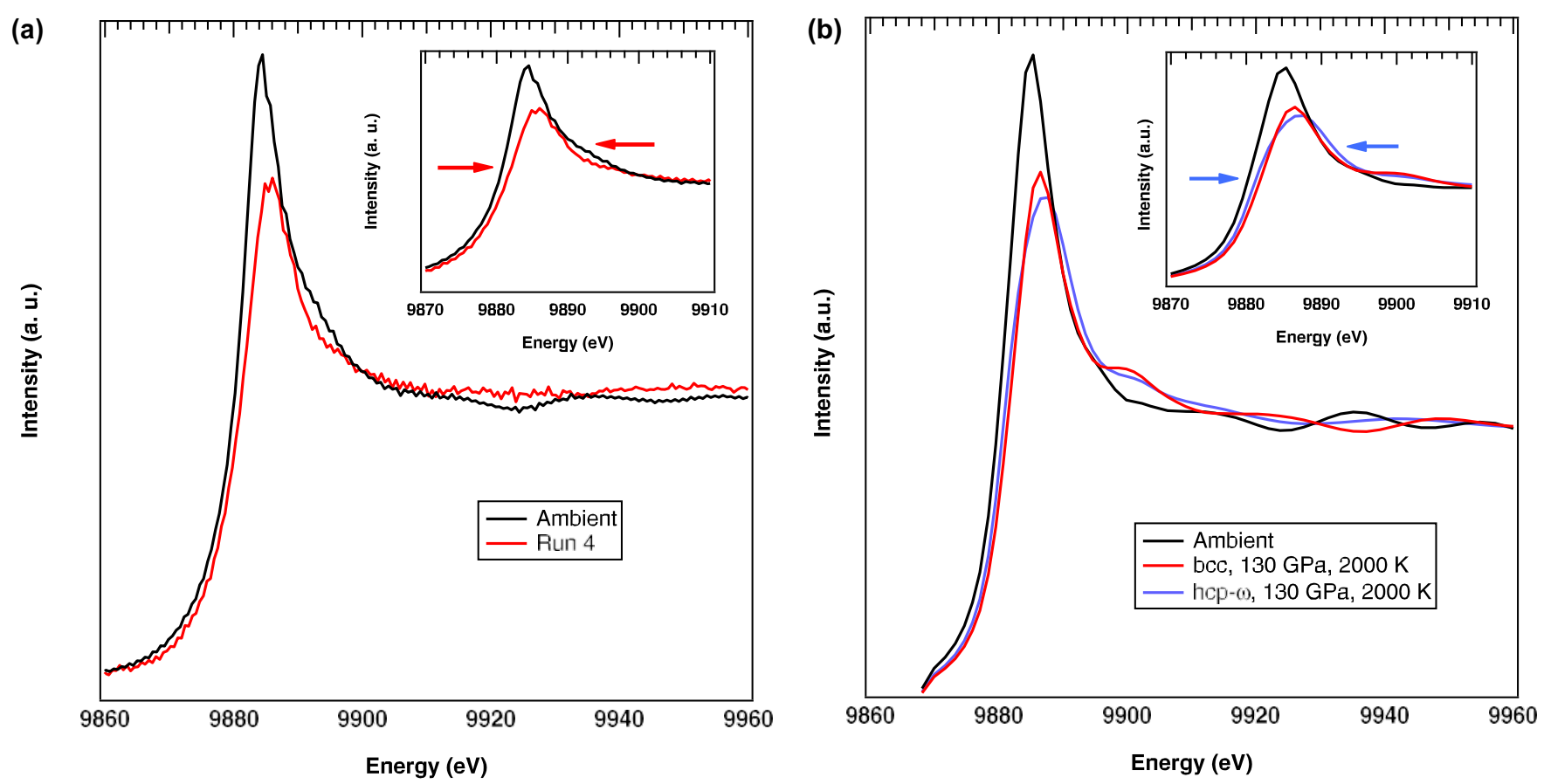

FIG. 7. (a) Experimental spectra of Ta near $L_{3}$ edge at ambient conditions and in run 4. (b) Comparison of XANES spectra for bcc and hcp- $\omega$ Ta using the FEFF software for the conditions estimated in the experiment. Insets: Focus on the XANES part. Arrows highlight the shape differences between the experimental (red) and calculated spectra for the $\omega$ phase (blue). The characteristic features of the $\omega$ phase, a broadening of the WL and a shoulder at $9882 \mathrm{eV}$, are not observed experimentally.

measurements. Conversely, $K$-edge spectroscopy is more appropriate for probing structural changes. Structural changes in Fe under shock compression had previously been followed by synchrotron $K$-edge XAS [21]. Here, the existence of the hcp- $\omega$ phase could only be questioned by looking at a change of the XANES spectra near the WL. No definitive answer could be obtained, only an indication that the spectra suggest the stability of the bcc phase. With the Extremely Brilliant Source (EBS) ESRF upgrade and the new beamline design that foresees the removal of the diamond window in the optics, an increase of the single-bunch intensity will bring a better signal-to-noise ratio, and probably the XANES spectra at the
$L$ edge could be analyzed as done previously at the $K$ edge. A definitive answer concerning the existence of the hcp- $\omega$ phase should probably be obtained.

\section{ACKNOWLEDGMENTS}

The authors acknowledge the European Synchrotron Radiation Facility for provision of synchrotron radiation on beamline ID24 (proposal HC2584). Lawrence Livermore National Laboratory is operated by Lawrence Livermore National Security, LLC, for the US Department of Energy, National Nuclear Security Administration, under Contract No. DE-AC52-07NA27344.
[1] D. Errandonea, B. Schwager, R. Ditz, C. Gessmann, R. Boehler, and M. Ross, Phys. Rev.B 63, 132104 (2001).

[2] D. Errandonea, M. Somayazulu, D. Haüsermann, and D. Mao, J. Phys.: Condens. Matter 15, 7635 (2003).

[3] A. Dewaele, M. Mezouar, N. Guignot, and P. Loubeyre, Phys. Rev. Lett. 104, 255701 (2010).

[4] A. Karandikar and R. Boehler, Phys. Rev.B 93, 054107 (2016).

[5] C. Dai, J. Hu, and H. Tan, J. Appl. Phys. 106, 043519 (2009).

[6] J. M. Brown and J. W. Shaner, in Shock Waves in Condensed Matter-1983, edited by J. R. Asay, R. A. Graham, and G. K. Straub (North-Holland, Amsterdam, 1984), p. 91.

[7] L. Burakovsky, S. P. Chen, D. L. Preston, A. B. Belonoshko, A. Rosengren, A. S. Mikhaylushkin, S. I. Simak, and J. A. Moriarty, Phys. Rev. Lett. 104, 255702 (2010).
[8] J. Hu, C. Dai, Y. Yu, Z. Liu, Y. Tan, X. Zhou, H. Tan, L. Cai, and Q. Wu, J. Appl. Phys. 111, 033511 (2012).

[9] L. M. Hsiung and D. H. Lassila, Scr. Mater. 38, 1371(1998).

[10] L. M. Hsiung and D. H. Lassila, Scr. Mater. 39, 603 (1998).

[11] L. M. Hsiung and D. H. Lassila, Acta Mater. 48, 4851 (2000).

[12] L. M. Hsiung, J. Phys.: Condens. Matter 22, 385702(2010).

[13] Y. Yao and D. D. Klug, Phys. Rev.B 88, 054102 (2013).

[14] Z.-L. Liu, L.-C. Cai, X.-L. Zhang, and F. Xi, J. Appl. Phys. 114, 073520 (2013).

[15] P. A. Rigg, R. J. Schar, and R. S. Hixson, J. Phys.: Conf. Ser. 500, 032018 (2014).

[16] F. Xi, K. Jin, L. Cai, H. Geng, Y. Tan, and J. Li, J. Appl. Phys. 117, 185901 (2015). 
[17] C. M. Pépin, A. Sollier, A. Marizy, F. Occelli, M. Sander, R. Torchio, and P. Loubeyre, Phys. Rev. B 100, 060101(R) (2019).

[18] S. Pascarelli, O. Mathon, T. Mairs, I. Kantor, G. Agostini, C. Strohm, S. Pasternak, F. Perrin, G. Berruyer, P. Chappelet, C. Clavel, and M. C. Dominguez, J. Synchrotron Radiat. 23, 353 (2016).

[19] O. Mathon, F. Occelli, E. Lescoute, A. Sollier, P. Loubeyre, W. Helsby, J. Headspith, R. Torchio, I. Kantor, and S. Pascarelli, High Press. Res. 36, 404 (2016).

[20] J. Headspith, J. Groves, P. N. Luke, M. Kogimtzis, G. Salvini, S. L. Thomas, R. C. Farrow, J. Evans, T. Rayment, J. S. Lee, W. D. Goward, M. Amman, O. Mathon, and S. Diaz-Moreno, IEEE Nucl. Sci. Symp. Conf. Rec. 4, 2421 (2007).

[21] R. Torchio, F. Occelli, O. Mathon, A. Sollier, E. Lescoute, L. Videau, T. Vinci, A. Benuzzi-Mounaix, J. Headspith, W. Helsby, S. Bland, D. Eakins, D. Chapman, S. Pascarelli, and P. Loubeyre, Sci. Rep. 6, 26402 (2016).

[22] J. P. Colombier, P. Combis, F. Bonneau, R. Le Harzic, and E. Audouard, Phys. Rev.B 71, 165406 (2005).

[23] R. Ramis, R. Schmalz, and J. Meyer-Ter-Vehn, Comput. Phys. Commun. 49, 475 (1988).

[24] The ABINIT code is a common project of the Université Catholique de Louvain, Corning Incorporated, the Commissariat à l'Energie Atomique, the Université de Liège, Mitsubishi Chemical Corp., and other contributors (http://www.abinit.org).

[25] X. Gonze, B. Amadon, P.-M. Anglade, J.-M. Beuken, F.Bottin, P. Boulanger, F. Bruneval, D. Caliste, R. Caracas, M. Côté et al., Comput. Phys. Commun. 180, 2582 (2009).

[26] F. Bottin, S. Leroux, A. Knyazev, and G. Zerah, Comput. Mater. Sci. 42, 329 (2008).

[27] X. Gonze, F. Jollet, F. Abreu Araujo, D. Adams, B. Amadon, T. Applencourt, C. Audouze, J.-M. Beuken, J. Bieder, A. Bokhanchuk et al., Comput. Phys. Commun. 205, 106 (2016).

[28] J. P. Perdew, K. Burke, and M. Ernzerhof, Phys. Rev. Lett. 77, 3865 (1996).

[29] P. E. Blöchl, Phys. Rev.B 41, 5414(R) (1990).

[30]
M. Torrent, F. Jollet, F. Bottin, and G. Zerah, Comput. Mater. Sci. 42, 337 (2008).

[31] M. Taillefumier, D. Cabaret, A.-M. Flank, and F. Mauri, Phys. Rev. B 66, 195107 (2002).

[32] N. A. W. Holzwarth, A. R. Tackett, and G. E. Matthews, Comput. Phys. Commun. 135, 329 (2001).

[33] M. Krauss and J. H. Oliver, J. Chem. Phys. Ref. Data 8, 329 (1979).

[34] P. Blaha, K. Schwarz, G. K. H. Madsen, D. Kvasnicka, and J. Luitz, WIEN2k, An augmented plane wave plus local orbitals program for calculating crystal properties, Vienna University of Technology, Austria, 2001, ISBN 3-9501031-1-2.

[35] K. Schwarz and P. Blaha, Comput. Mater. Sci. 28, 259 (2003).

[36] J. J. Rehr, J. J. Kas, F. D. Vila, M. P. Prange, and K. Jorissen, Phys. Chem. Chem. Phys. 12, 5503 (2010).

[37] J. J. Rehr and R. C. Albers, Rev. Mod. Phys. 72, 621 (2000).

[38] F. Dorchies and V. Recoules, Phys. Rep. 657, 1 (2016).

[39] A. Mančić, A. Lévy, M. Harmand, M. Nakatsutsumi, P. Antici, P. Audebert, P. Combis, S. Fourmaux, S. Mazevet, O. Peyrusse, V.Recoules, P. Renaudin, J. Robiche, F. Dorchies, and J. Fuchs, Phys. Rev. Lett. 104, 035002 (2010).

[40] A. Denoeud, S. Mazevet, F. Guyot, F. Dorchies, J. Gaudin, A. Ravasio, E. Brambrink, and A. Benuzzi-Mounaix, Phys. Rev.E 94, 031201(R) (2016).

[41] K. Oguri, Y. Okano, T. Nishikawa, and H. Nakano, Phys. Rev. Lett. 99, 165003 (2007).

[42] B. I. Cho, K. Engelhorn, A. A. Correa, T. Ogitsu, C. P. Weber, H. J. Lee, J. Feng, P. A. Ni, Y. Ping, A. J. Nelson, D. Prendergast, R. W. Lee, R. W. Falcone, and P. A. Heimann, Phys. Rev. Lett. 106, 167601 (2011).

[43] F. Dorchies, F. Festa, V. Recoules, O. Peyrusse, A. BenuzziMounaix, E. Brambrink, A. Lévy, A. Ravasio, M. Koenig, T. Hall, and S. Mazevet, Phys. Rev. B 92, 085117 (2015).

[44] E. Sevillano, H. Meuth, and J. J. Rehr, Phys. Rev. B 20, 4908 (1979).

[45] F. D. Vila, J. J. Rehr, H. H. Rossner, and H. J. Krappe, Phys. Rev. B 76, 014301 (2007).

[46] P. S. P. Wei and F. W. Lytle, Phys. Rev.B 19, 679 (1979). 